\title{
Crioconservación seminal de Colossoma macropomum como estrategia de producción y conservación en la Orinoquia Colombiana
}

\author{
Seminal cryopreservation of Colossoma macropomum as a production \\ and conservation strategy in the Colombian Orinoquia
}
Criopreservação seminal de Colossoma macropomum como estratégia de produção e conservação na Orinoquia Colombiana

\author{
Victor M. Medina-Robles ${ }^{1}{ }^{*}$; Diana N. Guaje-Ramírez ${ }^{2}$; Laura C. Marin-Cossio $^{2}$ *; \\ Leydy Y. Sandoval-Vargas ${ }^{3}$; Pablo E. Cruz-Casallas ${ }^{*}$ \\ 1 MVZ, MSc, cPhD; \\ 2 MVZ; \\ 3 Ing. Pecuária, cPhD; \\ 4 MVZ, Esp, MSc, PhD \\ * Grupo de Investigación sobre Reproducción y Toxicología de Organismos Acuáticos - GRITOX, Instituto de Acuicultura de \\ los Llanos - IALL, Facultad de Ciencias Agropecuarias y Recursos Naturales, Universidad de los Llanos, Villavicencio, Meta \\ - Colombia \\ Email:vmmedinarobles@unillanos.edu.co
}

Recibido:15 de octubre de 2018

Aprobado:31 de mayo de 2019

\begin{abstract}
Resumen
La Cachama negra (Colossoma macropomum) es un pez nativo de Sur América. En Colombia, no hay estudios publicados sobre protocolos estandarizados para su crioconservación seminal. La implementación de esta biotecnología permitiría su producción comercial continua e introducción en bancos de recursos genéticos. El objetivo del presente estudio fue evaluar los efectos de la crioconservación sobre el semen de C. macropomum sometido a diferentes crioprotectores y sistemas de empaque con miras a consolidar un protocolo eficiente para la especie. Se utilizó semen de tres machos sexualmente maduros (4.6 $\pm 1.6 \mathrm{~kg})$. El semen fue diluido en una proporción 1:4 usando tres diferentes agentes crioprotectores (Dimetilsulfoxido 10\% [DMSO], Metanol 10\% [MET], Etilenglicol 5\% [ETG]) con o sin la inclusión de yema de huevo 12\% (YH) y glucosa 5.5\%. Además, fueron evaluados dos sistemas de empaque (pajillas de $0.5 \mathrm{ml}$ y macrotubos de $2.0 \mathrm{ml}$ ), las cuales fueron expuestas a vapores de nitrógeno líquido (NL) y luego almacenadas durante 8 meses. El semen fue descongelado en baño de agua a $37^{\circ} \mathrm{C}$ por $60 \mathrm{~s}$ y se determinó la motilidad masal (\%) [MM], duración de la motilidad (s) [DM], integridad de membrana plasmática (\%) [IMP] y fertilidad (\%). La motilidad postdescongelación en todos los tratamientos fue significativamente diferente $(\mathrm{P}<0,05)$ al control siendo MET $2.0 \mathrm{ml}$ el mejor $(53 \pm 5.8 \%)$. Respecto al control la DM tuvo un comportamiento similar para todos los tratamientos siendo solo diferente significativamente para ETG+YH 0.5 ml. La IMP se mantuvo sin diferencias significativas en MET $2.0 \mathrm{ml}$ con respecto al control. La fertilidad fue significativamente menor en la mayoría de tratamientos con YH, siendo MET $2.0 \mathrm{ml}$ el mejor (94.7 $\pm 0.6 \%$ ). En conclusión, el semen de Colossoma macropomum es susceptible de crioconservación no siendo necesaria la utilización de YH en los diluyentes.
\end{abstract}


Palabras clave: criopreservación; peces nativos; semen; tambaquí.

\begin{abstract}
Cachama Negra (Colossoma macropomum) it's a native fish of South America. In Colombia, there are no published studies on standardized protocols about their seminal cryopreservation. The implementation of this biotechnology would allow its continuous commercial production and introduction in genetic resources banks. The aim of this study was to evaluate the effects of cryopreservation on C. macropomum sperm subjected to different cryoprotectants and packaging systems in order to consolidate an efficient protocol for this specie. Semen from three sexually mature males $(4.6 \pm 1.6 \mathrm{~kg})$ was used. The semen was diluted in a 1:4 ratio using three different cryoprotective agents (Dimethylsulfoxide 10\% [DMSO], Methanol 10\% [MET], Ethylene glycol 5\% [ETG]); with or without the inclusion of $12 \%$ egg yolk (YH) and glucose $5.5 \%$. In addition, two packing systems were evaluated $(0.5 \mathrm{ml}$ straws and $2.0 \mathrm{ml}$ macrotubes), wich were exposed to liquid nitrogen $(\mathrm{NL})$ vapors and then stored for 8 months. The sperm was thawed in a water bath at $37^{\circ} \mathrm{C}$ for $60 \mathrm{~s}$ and was determined the mass motility (\%) [MM], motility duration (s) [DM], plasma membrane integrity (\%) [IMP] and fertility (\%). Post-thaw motility in all treatments was significantly different $(\mathrm{P}<0.05)$ to control, being MET $2.0 \mathrm{ml}$ the best $(53 \pm 5.8 \%)$. Respect to the control the DM had a similar behavior for all treatments, being only significantly different for ETG+YH $0.5 \mathrm{ml}$. The IMP remained without significant differences in MET $2.0 \mathrm{ml}$ with respect to the control. Fertility was significantly lower in the majority of treatments with $\mathrm{YH}$, being MET $2.0 \mathrm{ml}$ the best $(94.7 \pm 0.6 \%)$. In conclusion, semen of Colossoma macropomum is susceptible to cryopreservation, and the use of $\mathrm{YH}$ in diluents is not necessary.
\end{abstract}

Key words: cryopreservation; native fish; semen; tambaqui.

\title{
Resumo
}

O tambaqui (Colossoma macropomum) é um peixe nativo da América do Sul. Na Colômbia, não há trabalhos publicados sobre protocolos padronizados para sua criopreservação seminal. A implementação dessa biotecnologia permitiria sua produção comercial contínua e sua introdução nos bancos de genes. O objetivo deste estudo foi avaliar os efeitos da criopreservação no sêmen de C. macropomum submetido a diferentes crioprotectores e envases, e poder consolidar um protocolo eficiente para esta espécie. Foi utilizado sêmen de três machos sexualmente maduros (4.6 $\pm 1.6 \mathrm{~kg})$. O sémen foi diluído em uma proporção 1:4 usando três diferentes agentes crioprotectores (10\% de dimetil sulfóxido [DMSO], 10\% de Metanol [MET] Etileno glicol 5\% [ETG]), com e sem a presencia de gema de ovo ao 12\% (YH) e glucose a 5.5\%. Além foram avaliados dos sistemas de envase (palhetas de $0.5 \mathrm{ml}$ e $2.0 \mathrm{ml}$ de macrotubos) que foram expostos a vapores de nitrogênio líquido (NL) e armazenados por 8 meses em NL. O sémen foi descongelado em banho-água a $37^{\circ} \mathrm{C}$ durante 60 segundos e determinou-se a motilidade massal (\%) [MM], duração de motilidade (s) [DM], a integridade da membrana plasmática (\%) [IMP] e Fertilidade (\%). A motilidade pós-descongelação nos tratamentos foi significativamente diferente $(\mathrm{P}<0.05)$ sendo a melhor combinação MET $2.0 \mathrm{ml}(53 \pm 5.8 \%)$ comprados com o controle. Em relação ao controle, o DM teve um comportamento similar para todos os tratamentos sendo significativamente diferente para o ETG+YH $0.5 \mathrm{ml}$. O IMP permaneceu sem diferenças significativas no MET $2.0 \mathrm{ml}$ em relação ao controle. A fertilidade foi significativamente menor na maioria dos tratamentos com YH, sendo o MET $2.0 \mathrm{ml}$ o melhor (94.7 $\pm 0.6 \%$ ). Em conclusão, o sêmen Colossoma macropomum é suscetível à criopreservação e o uso de YH em diluentes não é necessário.

Palavras chave: criopreservação; peixe nativo; sêmen; tambaqui.

\section{Introducción}

La cachama negra (Colossoma macropomum) es un pez perteneciente a la familia de los carácidos, nativo de las cuencas del Amazonas y del Orinoco donde representa un importante producto pesquero (Castillo, 2005; FAO, 2015). Según Sanabria (2004) en Colombia se localiza en los ríos Amazonas, Putumayo, Caquetá, Orinoco y Meta; en aguas con temperaturas de 22 a $28{ }^{\circ} \mathrm{C}$ y $\mathrm{pH}$ de 5.0 a 7.8 .

De acuerdo a la FAO (2015) en Colombia en el periodo 2013 y 2014, C. macropomum se identificó dentro de los grupos de especies de peces más representativos en cuanto a volúmenes totales (90863 toneladas aproximadamente) desembarcados en la cuenca del Orinoco, aportando el 5\% (605 toneladas) del total de la contribución. Sin embargo, se sitúa por debajo del grupo de los bagres (especies no especificadas) que aportaron el 45\% (4959 toneladas) y al bocachico (Prochilodus mariae) con una contribución del 20\% (2218 toneladas). En la cuenca del Amazonas en este mismo periodo con un estimado de 56165 toneladas desembarcadas, C. macropomum aportó el 0.3\% (168 toneladas), igualmente por debajo de los bagres que aportaron el 57\% (32003 toneladas) y bocachico (Prochilodus nigricans) con una contribución del 1.1\% (622 toneladas).

Millones de personas en todo el mundo encuentran una fuente de ingresos y medios de vida en los sectores de la pesca y la acuicultura. Estadísticas oficiales indican que 59,6 millones de personas que participa- 
ron en el sector primario de la pesca de captura y la acuicultura en 2016, 19,3 millones lo hicieron en la acuicultura y 40,3 millones en la pesca. La proporción de personas que se dedicaban a la pesca de captura disminuyó del 83\% en 1990 al 68\% en 2016, mientras que la de las personas que se dedicaban a la acuicultura aumentó en consecuencia del 17\% al 32\% (FAO, 2018). A nivel Nacional Merino et al., (2013) describen la tendencia de crecimiento exponencial de la acuicultura en Colombia en el período 1990 - 2011, al pasar de 9200 toneladas en 1990 a 82733 en 2011 y casi 110000 toneladas en 2015, es decir, un $12 \%$ anual promedio $y$, aunque muestra una menor aceleración que otros países de Latinoamérica, supera por mucho la tasa media del crecimiento del resto del sector agropecuario y del conjunto total de la economía nacional.

Por otra parte, a pesar del crecimiento de la acuicultura, el cambio climático es un fenómeno que causa consecuencias a corto, mediano y largo plazo, pudiendo conllevar a efectos negativos en los procesos de reproducción y conservación de las especies. Da Silva y Soto (2009) exponen algunos factores que podrían repercutir potencialmente en la producción de peces tales como, la subida del nivel del mar, el aumento de la temperatura, cambios en los patrones de pluviosidad y el estrés hídrico, variando de acuerdo a las distintas zonas climáticas. Los peces por ser organismos ecotérmicos, presentan alta sensibilidad a cambios de la temperatura del ambiente y del agua, reflejándose de igual forma, en alteraciones del comportamiento, crecimiento e incluso su reproducción, lo que conllevaría posiblemente a un acortamiento de la temporada reproductiva y alterando las condiciones óptimas para el desarrollo de la progenie (Miranda et al., 2013).

Por las razones antes expuestas, autores como Poleo et al., (2011) postulan que en la medida que la actividad acuícola y la creciente demanda de fuentes de proteína animal se incrementen, se presenta la necesidad de diversificar los modos de producción. Por lo anterior, la utilización de biotecnologías como la crioconservación seminal son cada vez más importantes, dado que, contribuyen no solo a suplir las necesidades de producción continua de alimentos como fuente de proteína animal y mantener la seguridad alimentaria, sino que también, permiten conservar, proteger y mantener el material genético de especies nativas con potencial económico y ambiental, a través del establecimiento de bancos de germoplasma.

Sumado a esto, la crioconservación de semen en peces, como en otras especies, es una biotecnología con varias variables por consolidar, las cuales tienen efectos sobre la calidad espermática comprometiendo directamente la capacidad de la célula para los procesos de fertilización y desarrollo embrionario (María et al., 2006). Por esta razón, es necesario el perfeccionamiento de técnicas que aborden la evaluación de los factores implicados en el proceso de crioconservación y que permitan definir protocolos sencillos y efectivos para la producción continua y conservación de la especie.

Por lo anterior, el presente trabajo evaluó tres diferentes agentes crioprotectores, dos sistemas de empaque y su efecto sobre la calidad postdescongelación del semen de cachama negra (Colosoma macropomum) sometido a procesos de crioconservación.

\section{Materiales y métodos}

\section{Localización o área de estudio}

El semen fue colectado de machos adultos maduros pertenecientes a la granja piscícola Langostinos del Llano ubicada en el municipio de Restrepo - Meta a una altitud promedio de $570 \mathrm{msnm}$, temperatura de $25.8^{\circ} \mathrm{C}$, precipitación pluvial de $5062 \mathrm{~mm}$ y humedad relativa del $83 \%$. Allí se determinaron las características seminales del semen fresco y se realizó la crioconservación seminal. Luego de esto, el material genético congelado fue llevado al Laboratorio de Reproducción de Peces y Crioconservación del Instituto de Acuicultura de la Universidad de los Llanos - IALL, localizado a $12 \mathrm{Km}$ de la ciudad de Villavicencio - Meta. Este instituto se encuentra a una altura promedio de 418 msnm, temperatura de $25^{\circ} \mathrm{C}$, precipitación pluvial de $4050 \mathrm{~mm}$ y humedad relativa del $75 \%$.

\section{Material biológico}

Se utilizaron tres machos $(n=3)$ de cachama negra (Colossoma macropomum) sexualmente maduros (4.6 $\pm 1.6 \mathrm{~kg}$ ) de acuerdo a la disponibilidad de animales en la granja piscícola, inducidos mediante una dosis única $(4 \mathrm{mg} / \mathrm{kg}$ ) de extracto de pituitaria de carpa (EPC) y espermiados a las 20 horas de aplicación. Tras ser anestesiados los reproductores con Fenoxietanol (300ppm), el semen se extrajo por medio de masaje cráneo-caudal sobre la región ventral y colectado en tubos de vidrio aforados para determinar el volumen seminal (VS) y sus características macroscópicas (color). Luego de esto, se evaluaron mediante microscopio óptico (Carl Zeiss, Alemania) la motilidad masal (MM), duración de la motilidad (DM), concentración espermática (CE), integridad de membrana plasmática (IMP) y morfología espermática (ME). 
Posteriormente, se realizó un pool de semen el cual fue diluido en proporción 1:4 (semen/diluyente) en una solución base de glucosa-5.5\% (p:v Merck Schuchardt OHG., Hohenbrunn, Germany). Se utilizaron como crioprotectores permeables Dimetil-sulfoxido DMSO 10\% (v:v Merck Schuchardt OHG., Hohenbrunn, Germany), Metanol MET 10\% (v:v Merck Schuchardt OHG., Hohenbrunn, Germany) y Etilenglicol ETG 5\% (v:v Merck Schuchardt OHG., Hohenbrunn, Germany), haciendo uso o exclusión de Yema de Huevo-12\% (YH) como crioprotector externo. El semen diluido fue empacado en pajillas de $0.5 \mathrm{ml}$ y macrotubos de $2.0 \mathrm{ml}$ y congelados en vapores de nitrógeno líquido por medio de un Dry Shipper (DS). Ocho meses después el semen fue descongelado en baño de agua a $37^{\circ} \mathrm{C}$ por $60 \mathrm{~s}$ y evaluadas las variables MM, DM, IMP y ME postdescongelación. Como control se utilizó semen fresco.

\section{Análisis espermáticos}

La MM fue determinada de manera subjetiva, de acuerdo a los protocolos estandarizados en el grupo de investigación y determinada por la misma persona. Esto fue realizado activando una alícuota de semen $(20 \mu \mathrm{L})$ con $180 \mu \mathrm{L}$ de agua destilada $(1: 10)$ y haciendo uso de una lámina de vidrio excavada (1.0 - 1.2 $\mathrm{mm}$ de profundidad, Micro Slides Premiere, China) ubicada en un microscopio óptico (Zeiss, Alemania) a 10X de magnificación. La MM se definió como el porcentaje (\%) de motilidad o movimiento en masa de la muestra de semen y la DM fue definida como el intervalo de tiempo en segundos (s) entre la activación espermática y la perdida de movimiento superior al $90 \%$ de la muestra.

La CE se calculó mediante el recuento de espermatozoides en una cámara de Neubauer y fue expresada en $\mathrm{spz} / \mu \mathrm{L} .10^{6}$. Las variables en semen fresco fueron determinadas para cada uno de los tres animales con el objetivo de tener una media de los datos. Para semen crioconservado la pajilla $(n=3)$ fue considerada como la repetición.

Para IMP se realizaron extendidos utilizando $5 \mu \mathrm{L}$ de semen fresco y $45 \mu \mathrm{L}(1: 10)$ de la solución eosina $1.1 \%$ - nigrosina 5.3\% - citrato de sodio 2.9\% sobre lámina portaobjeto (Microscope slides, Ground edges) y observado mediante microscopio óptico de contraste (Nikon- Eclipse E-400, Japón) a un aumento de 40x. Se determinaron espermatozoides con membrana plasmática intacta aquellos que permanecieron traslucidos y como espermatozoides con ruptura de membrana plasmática aquellos que presentan una tonalidad morada debido a la permeabilidad de la tinción. La IMP fue evaluada sobre 100 espermatozoides en cada muestra y se expresó como porcentaje \% de IMP.

Para el caso de la ME, una muestra de $1 \mu \mathrm{L}$ de semen en fresco fue fijada en $999 \mu \mathrm{L}$ de solución formol - citrato [formaldehído 35\% (4\%) y citrato de sodio (2.9\%)] en un tubo Eppendorff (1:1000). En el momento del análisis, se colocaron $15 \mu \mathrm{L}$ de esta mezcla homogenizada mediante un Vórtex (Fisher Scientific ${ }^{\mathrm{TM}}$ Digital Vortex Mixer, EEUU), sobre una lámina portaobjeto (Microscope slides, Ground edges) y seguido de esto se añadieron $0.5 \mu \mathrm{L}$ de Rosa de Bengala 3\%; se mezcló y se realizó el extendido.

El análisis morfológico se realizó sobre 100 espermatozoides mediante microscopio óptico de contraste de fase (Nikon- Eclipse E-400, Japón) a una magnificación de 1000X, donde se determinó el número de espermatozoides normales y el número de espermatozoides con anormalidades morfológicas como: macrocefalia, cabeza separada, cabeza degenerada, degeneración pieza media, gota citoplasmática proximal, cola separada, cola doblada, cola rota, cola en espiral o cola corta. Los resultados de morfología espermática fueron expresados como \% de anormalidades.

La MM, DM, IMP y la ME del semen postdescongelación fueron determinadas siguiendo el mismo procedimiento utilizado con el semen fresco. El \% de Anormalidades para el tratamiento de ETG en pajillas de $0.5 \mathrm{ml}$ no fue determinado por ruptura de las pajillas destinadas para este fin durante el proceso de crioconservación.

\section{Pruebas de fertilidad}

Para la determinación del \% de fertilidad, las pajillas fueron descongeladas a $37^{\circ} \mathrm{C}$ durante 60 segundos. Luego de esto se determinó la motilidad y duración de la motilidad utilizando los mismos protocolos del semen fresco. El semen fue activado con una solución de bicarbonato de sodio $\left(\mathrm{NaHCO}_{3}\right)$ al $1 \%$ ya que esta solución prolonga la duración de la movilidad de espermatozoides descongelados según lo reportado por Ramírez-Merlano et al., (2011).

Se utilizaron 2 hembras maduras (5.5 $\pm 0.49 \mathrm{~kg})$, las cuales fueron inducidas mediante EPC a dosis total de $5.75 \mathrm{mg} / \mathrm{kg}$ de peso corporal, dividida en tres inyecciones (0, 24 y 36 horas), el desove se realizó 6 horas después de la última aplicación de hormona. Para la prueba de fertilidad se utilizaron 2 gr de oocitos/ tratamiento del pool de las dos hembras, lo cual fue necesario para lograr la cantidad de oocitos requeridos para el número de réplicas estadísticamente establecido para la prueba de fertilidad. Seguido de esto, 
se realizó la fertilización de los oocitos con $100 \mu \mathrm{L}$ y $400 \mu \mathrm{L}$ de semen fresco y crioconservado, respectivamente, con el objetivo de mantener un equilibrio en el número de espermatozoides viables por oocito. La incubación se realizó en recipientes cónicos de plástico con aireación constante y la fertilidad fue determinada seis horas después de la fertilización de los oocitos. La fertilidad se expresó en porcentaje y fue calculada mediante la proporción de oocitos fertilizados (traslucidos) versus aquellos no viables (blancos) sobre un total de 50 oocitos contados, realizando dicho procedimiento tres veces por tratamiento.

\section{Análisis estadístico}

Los datos se presentan como media \pm SEM. Se usó estadística descriptiva y el respectivo análisis paramétrico de los datos por medio ANOVA y post test de Tukey para comparación de medias. Previamente, se verificaron los supuestos de normalidad (KolmogorovSimirnov) y homogeneidad de varianzas (Bartlett). El nivel de significancia aceptado fue de $\mathrm{P}<0.05$. Los análisis estadísticos fueron realizados usando los paquetes de RStudio ${ }^{\circledR}$ y Rstadistics 3.2.2®.

\section{Resultados}

Los resultados obtenidos de la evaluación de las características macroscópicas y microscópicas del semen fresco se muestran en la Tabla 1.

Tabla 1. Parámetros en semen fresco de Colossoma macropomum. Valores mostrados como Media \pm SEM. $(n=3)$

\begin{tabular}{|l|c|}
\hline \multicolumn{1}{|c|}{ Parámetro } & $\begin{array}{c}\text { Media } \pm \\
\text { SEM }\end{array}$ \\
\hline Volumen seminal $(\mathrm{ml})$ & $3 \pm 2$ \\
\hline Concentración espermática $\left(\mathrm{spz} / \mu \mathrm{L} 10^{6}\right)$ & $4.5 \pm 4.3$ \\
\hline Motilidad masal $(\%)$ & $95 \pm 0.0$ \\
\hline Viabilidad espermática $(\%)$ & $88 \pm 6.1$ \\
\hline Anormalidades $(\%)$ & $25 \pm 4.5$ \\
\hline
\end{tabular}

En cuanto a la motilidad (Figura 1) y \% de anormalidades postdescongelación (Figura 2) todos los tratamientos fueron significativamente diferentes $(\mathrm{P}<0.05)$ al control, siendo el tratamiento con MET 10\% empacado en macrotubos de $2.0 \mathrm{ml}$ el que obtuvo mejores resultados para motilidad $(53 \pm 5.8 \%$ ) y diferente significativamente $(\mathrm{P}<0.05)$ comparado con los demás tratamientos. Este mismo, además presentó menor \% de anormalidades espermáticas $(61.3 \pm 5.1 \%)$ postdes-

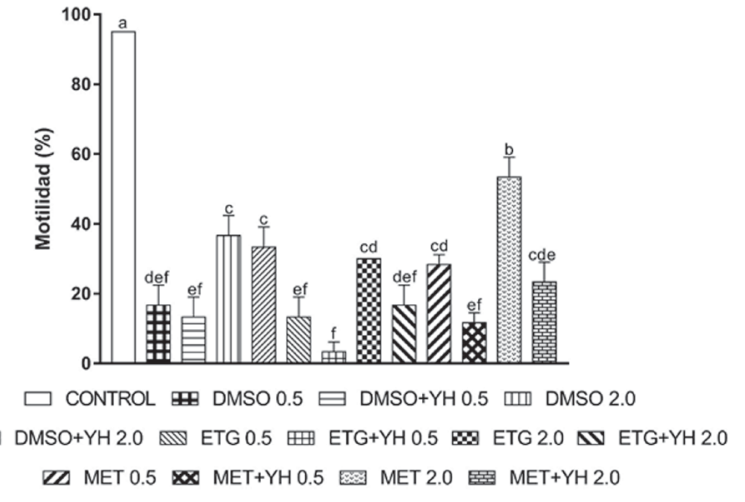

Figura 1. Motilidad post-descongelación de semen de Colossoma macropomum. Control= semen fresco. Entre barras letras distintas indican diferencia estadística $(P<0.05)$. Valores mostrados como media \pm SEM. $n=3$

congelación. En el caso de ETG+YH $0.5 \mathrm{ml}$ fue el tratamiento que mostró los resultados más desfavorables, obteniendo el \% de motilidad más bajo y mayor \% de anormalidades $(3.3 \pm 2.9 \%$ y $95.7 \pm 5.1 \%$, respectivamente)

Para el caso del \% de IMP postdescongelación, se observó que todos los tratamientos tuvieron diferencia significativa $(\mathrm{P}<0.05)$ con respecto al semen fresco a excepción de MET $2.0 \mathrm{ml}(83 \pm 1.5 \%)$ quien no tuvo diferencia significativa. Sin embargo, también se determinó que el uso de ETG y MET+YH empacados en macrotubos de $2.0 \mathrm{ml}$ a pesar de que tuvieron diferencia significativa $(\mathrm{P}<0.05)(81 \pm 1.2 \%$ y $80 \pm 4 \%$, respectivamente) con respecto al semen fresco, mostraron valores cercanos a este mismo $(97 \pm 1.5)$. Los porcentajes

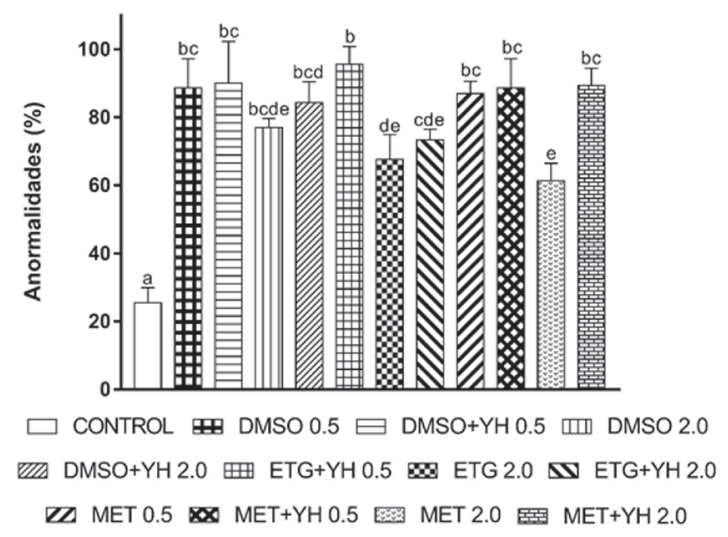

Figura 2. Anormalidades espermáticas post-descongelación de semen de Colossoma macropomum. Control= semen fresco. Entre barras letras distintas indican diferencia estadística $(\mathrm{P}<0.05)$. Los valores para ETG $0.5 \mathrm{ml}$ no se determinaron. Valores mostrados como media \pm SEM. ( $n=100$ espermatozoides/tratamiento) 
de integridad de membrana plasmática más bajos se obtuvieron mediante el uso de ETG $0.5 \mathrm{ml}$ y ETG+YH $0.5 \mathrm{ml}(49.67 \pm 0.88$ y $41.33 \pm 5.0$, respectivamente $)$ (Figura 3).

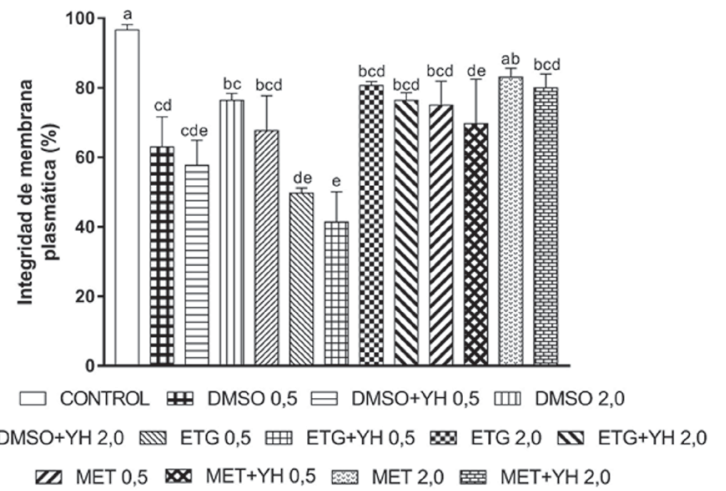

Figura 3. Integridad de membrana plasmática post-descongelación de semen de Colossoma macropomum. Control= semen fresco. Entre barras letras distintas indican diferencia estadística $(P<0.05)$. Valores mostrados como media \pm SEM. ( $n=100$ espermatozoides/tratamiento)

Con relación con el \% de anormalidades espermáticas postdescongelación, la que más se presentó fue la cola doblada siendo mayor en el tratamiento DMSO $0.5 \mathrm{ml}$ $(61.3 \pm 8.1 \%)$ y menor en el tratamiento MET+YH 0.5 $\mathrm{ml}(21 \pm 10 \%)$ respecto al control (18.8 $\pm 7.0 \%)$. Por otra parte, se observó que la presentación de macrocefalia en los espermatozoides no fue significativa (Tabla 2). Las anormalidades morfológicas se presentaron en todos los tratamientos y algunas de las alteraciones observadas microscópicamente se muestran en la Figura 4.

La fertilidad fue significativamente menor en la mayoría de tratamientos a base de $\mathrm{YH}$ (Figura 5), siendo MET $2.0 \mathrm{ml}$ el mejor tratamiento, ya que presentó el mayor porcentaje de fertilidad $(94.7 \pm 0.6 \%)$ con respecto al control $(96.7 \pm 1.1 \%)$. El tratamiento que obtuvo el menor porcentaje de fertilidad fue ETG+YH 0.5 $\mathrm{ml}$ con un valor de $49.7 \pm 1.5 \%$

\section{Discusión}

En Colombia no existen estudios publicados acerca del proceso de crioconservación seminal de Colossoma macropomum, siendo el presente estudio una base de conocimiento para la consolidación de un paquete tecnológico basado en la biotecnología de crioconservación, susceptible de ser transferida a los piscicultores e incluida dentro de los procesos de establecimiento de un banco de germoplasma de especies ícticas nativas para la Orinoquia Colombiana.
Con respecto a la calidad seminal determinada, estudios realizados en otros países reportan un VS en machos inducidos $(5.05 \pm 2.0 \mathrm{ml})$ similar a los resultados obtenidos en el presente trabajo y superiores en cuanto a CE $\left(22,93 \pm 9,79 \times 10^{9} \mathrm{sptz} / \mathrm{ml}\right)$ (Vieira et al., 2011). De igual forma, María et al., (2010) determinaron VS en la especie con valores muy superiores (10.2 $\pm 5.1 \mathrm{ml}$ ) a los encontrados en el presente estudio. El volumen seminal en trabajos de crioconservación se convierte en una variable de gran importancia, ya que permite proyectar los posibles sistemas de empaque para el semen, siendo recomendable en especies de alta fecundidad el empaquetamiento en macrotubos, para optimizar la eficiencia en el proceso de descongelación y seminación.

Con respecto a la evaluación de la motilidad postdescongelación, no fue observado un comportamiento homogéneo en el semen diluido y empacado en pajillas de 0.5 y $2.0 \mathrm{ml}$. Lo anterior, indica que el sistema de empaque podría tener incidencia en los resultados de motilidad postdescongelación para la especie de estudio como ha sido observado en otros trabajos (Medina et al., 2007; Ramírez-Merlano et al., 2011). Esto contrasta con los estudios realizados por María et al., (2015), donde se evaluaron empaques con diferentes volúmenes $(1.6$ y $4.5 \mathrm{ml})$ sin diferencias significativas para esta misma característica seminal. La determinación de las tasas de congelación/descongelación y su asociación con el sistema de empaque, se convierte en una variable importante de establecer en futuros trabajos para dar mayor explicación a los resultados encontrados.

María et al., (2015) determinaron porcentajes de IMP (viabilidad) postdescongelación en dos diferentes tiempos de descongelación (70 y 90 s) de $46 \pm 10$ y $54 \pm 9 \%$, respectivamente, mediante el uso de metilglicol como crioprotector. Esto comparado con los resultados obtenidos en el presente trabajo, muestra valores similares con el uso de ETG+YH $0.5 \mathrm{ml}$ (41 \pm $8.7 \%$ ), mientras que el uso de MET $2.0 \mathrm{ml}$ obtuvo valores superiores a éstos (83 $\pm 2.6 \%$ ). María et al., (2011) afirman que una muestra de buena calidad de semen descongelado es aquella que presenta motilidad y viabilidad superior al 50\%; valores que fueron superados por algunos de los tratamientos utilizados en el presente estudio. En contraste, el trabajo realizado por Ramírez et al., (2011), demuestran por determinación de motilidad por análisis espermático computarizado, que no siempre es necesaria la motilidad progresiva para la fertilización en peces de fecundación externa como Pseudoplatystoma metaense, ya que muestras seminales crioconservadas cuya motilidad postdescongelación masal fue baja pero con altos porcentaje de 

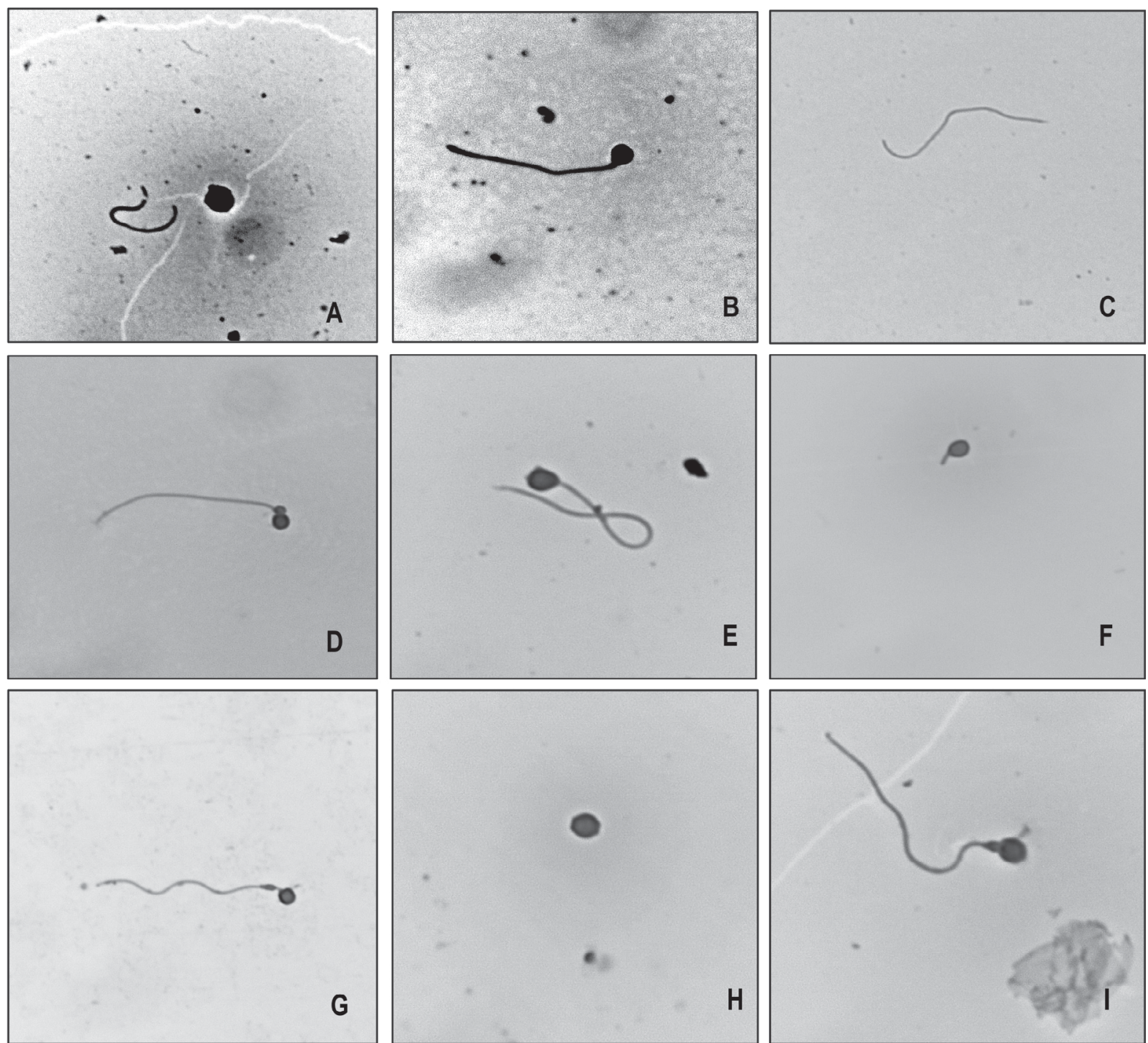

Figura 4. Anormalidades morfológicas en semen descongelado de cachama negra (Colossoma macropomum). A: cola rota, B macrocefalia, C cola separada, $\mathbf{D}$ gota citoplasmática, E cola doblada, $\mathbf{F}$ cola corta, $\mathbf{G}$ cola en espiral, $\mathbf{H}$ cabeza separada y I degeneración pieza media. Magnificación 1000x.

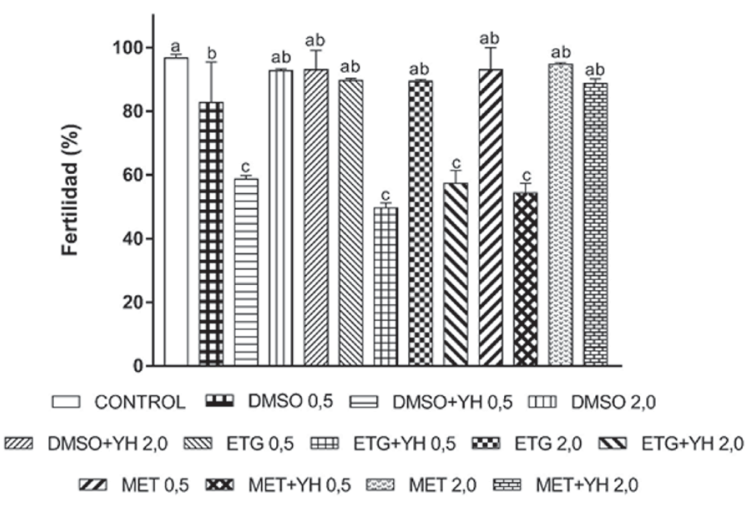

Figura 5. Parámetros de fertilidad en semen en fresco y congelado de Colossoma macropomum. Control= semen fresco. Entre barras letras distintas indican diferencia estadística $(P<0.05)$. Valores mostrados como media \pm SEM. $(n=3)$ motilidad local o en círculo mantuvieron intacta su capacidad fecundante, con porcentajes de fertilidad por encima del $60 \%$.

El proceso de crioconservación en el presente estudio ocasionó una pérdida significativa de la calidad del espermatozoide en cuanto a la morfología, debido posiblemente al estrés osmótico celular derivado del proceso de congelación/descongelación, a efectos de criodaño por procesos biofísicos como la cristalización o a posibles alteraciones de tipo iatrogénicas ocasionadas durante la manipulación de las muestras. Otras eventuales causas de aumento de anormalidades espermáticas postdescongelación han sido atribuidas al tipo de contenedor de almacenamiento y a la incorporación del diluyente con el semen (María et al., 2009). Oliveira et al., (2016) observaron que el 


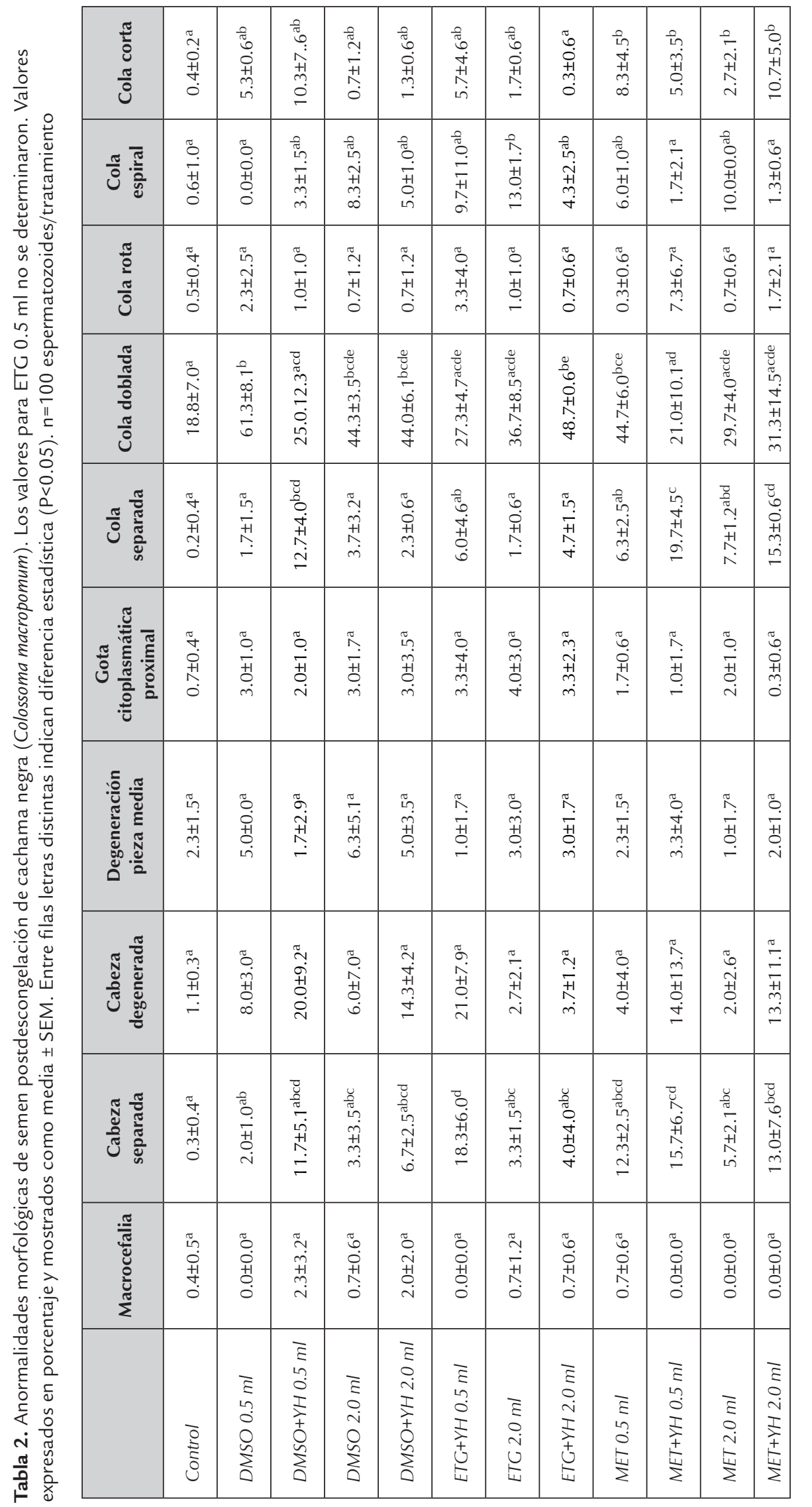

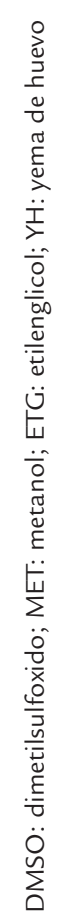


uso de equipos de congelación programada comparado con el uso de vapores de nitrógeno o sistemas "dry shipper", permitió mantener un mayor número de espermatozoides normales. Además, Espinoza et al., (2011) reportaron que la morfología espermática de Argopecten purpuratus fue afectada por el proceso de congelación/descongelación, comparado con el uso de crioprotectores sin sometimiento a crioconservación. Sin embargo, aunque todos los tratamientos en el presente estudio tuvieron anormalidades espermáticas significativamente superiores con respecto al semen fresco, todos presentaron fertilidad por encima del $60 \%$.

El desarrollo de pruebas de fertilidad dentro de los estudios de crioconservación son de suma importancia, ya que respaldan y permiten asociar los resultados de las demás características seminales posdescongelación. De igual forma, la evaluación de diluyentes sencillos con la incorporación o exclusión de sustancias crioprotectoras externas como la yema de huevo, también deben ser validados. En el presente trabajo no se observó diferencia significativa entre la mayoría de tratamientos, para todas las variables de calidad seminal haciendo uso o exclusión de YH, con excepción de los tratamientos congelados con MET en pajillas de 2.0 $\mathrm{ml}$, donde el uso de $\mathrm{YH}$ generó valores inferiores en cuanto a las variables de calidad seminal. Esto concuerda con estudios como el de Leite et al., (2012) donde probaron dos concentraciones de yema de huevo (5\% y $10 \%$ ) y, además la exclusión de ella en la crioconservación de semen de C. macropomum, concluyendo que al agregar este crioprotector externo se disminuye significativamente el porcentaje de espermatozoides móviles posdescongelación. Esto difiere con lo reportado por Carneiro et al., (2012) quienes encontraron que el uso de $\mathrm{YH}$ mejoró las características de calidad seminal posdescongelación. Sin embargo, sugiere reducir la adición de $\mathrm{YH}$ del $10 \%$ al 5\% como una alternativa para la congelación de semen obteniendo resultados de calidad seminal semejantes o superiores, siendo $10 \%$ una concentración de uso tradicional.

Estudios realizados por Junior et al., (2012), resaltan el uso de amidas como crioprotectores externos alternativos para la congelación de semen de C. macropomum, cuya efectividad posiblemente es debido a su actividad lipofilica, bajo peso molecular y baja viscosidad, lo cual les permite gran permeabilidad de la membrana plasmática, eficiencia en el anclaje de moléculas, reducción del estrés osmótico y disminución de la formación de cristales de hielo. Estos autores evaluaron la incorporación de Dimetilacetamida en el diluyente, obteniendo las mayores tasas de motilidad postdescongelación con concentraciones de 5, 8 y
$11 \%(55.7 \pm 2.4,64.3 \pm 3.0$ y $62.5 \pm 3.1 \%$, respectivamente). Crioprotectores externos similares podrían ser evaluados para optimizar el presente protocolo de congelación en la especie y mejorar la eficiencia en los porcentajes de motilidad postdescongelación y fertilidad.

Por otra parte, Varela et al., (2012) concluyen que la adición de DMSO a una concentración de 10\% se asoció con una mejor calidad del semen postdescongelación de Tambaquí, cuando comparado con concentraciones de 5, 15 y 20\%. No obstante, García et al., (2015) demuestran que el uso se soluciones extensoras combinadas con un $10 \%$ de DMSO, mantuvieron intacto el ADN del espermatozoide en casi todas las células espermáticas de C. macropomum, aun cuando hubo pérdida importante en su funcionalidad. Aunque en éste estudio no fue evaluada la integridad del ADN ni los efectos del tiempo de crioconservación sobre la calidad seminal postdescongelación y desarrollo larval, Calizaya y Yam (2013) reportan efectos negativos importantes sobre la progenie cuando comparado con semen fresco, variable que debe ser estudiada con fines de transferencia de la biotecnología a productores.

En conclusión, el uso de Metanol al 10\% y macrotubos de $2.0 \mathrm{ml}$ permiten preservar de manera adecuada el material seminal de Colossoma macropomum. A pesar del alto porcentaje de anormalidades espermáticas postdescongelación con respecto al semen fresco, se alcanzaron valores de motilidad postdescongelación y fertilidad aceptables para procesos de crioconservación en la especie, sin ser necesaria la inclusión de YH en los diluyentes. Se hace necesario realizar estudios adicionales con el fin de consolidar procedimientos de crioconservación de semen en la especie para su uso en sistemas productivos y para el establecimiento de bancos de germoplasma.

\section{Agradecimientos}

A la granja piscícola Langostinos del Llano por el suministro de los reproductores de la especie. El presente trabajo es apoyado por el Fondo Social de Educación Superior de la Gobernación del Meta a través de la beca de doctorado otorgada al autor Víctor Mauricio Medina Robles en la convocatoria de "Formación de capital humano de alto nivel 2015 - Gobernación del Meta".

\section{Referencias}

Calizaya L, Yam E. 2013. Efecto de diferentes tiempos de criopreservación sobre la viabilidad del semen de Colossoma macropo- 
mum" gamitana". (Tesis de maestría). Universidad Nacional de la Amazonía Peruana. Iquitos, Perú. 65 p

Castillo O. 2005. La piscicultura como alternativa de producción animal en Venezuela. Sistemas integrados de producción con no rumiantes. UNELLEZ. Portuguesa, Venezuela, 44-46.

Carneiro PC, Azevedo HC, Santos JP, María AN. Cryopreservation of tambaqui (Colossoma macropomum) semen: extenders, $\mathrm{cr}$ yoprotectants, dilution ratios and freezing methods. CryoLetters. 2012;33(5):385-393.

De Silva SS, Soto D. El cambio y la acuicultura: repercusiones potenciales, adaptación y mitigación. Consecuencias del cambio climático para la pesca y la acuicultura: visión de conjunto del estado actual de los conocimientos científicos. FAO Documento Técnico de Pesca y Acuicultura. 2009;(530):169-236.

Espinoza C, Valdivia M, Dupré E. Morphological alterations in cryopreserved spermatozoa of scallop Argopecten purpuratus. Lat Am J Aquat Res. 2011;38(1):123-130.

FAO - Food and Agriculture Organization (2015). Colombia. Pesca en cifras 2014. Bogotá, Colombia.

FAO - Food and Agriculture Organization (2018). El estado mundial de la pesca y la acuicultura 2016. Cumplir los objetivos de desarrollo sostenible. Roma. 233 pp.

García RRF, Vasconcelos ACN, Povh JA, Oberst ER, Varela Jr AS, Corcini CD, Streit Jr DP. Functional integrity of Colossoma macropomum (Cuvier, 1816) sperm cryopreserved with enriched extender solutions. Neotrop Ichthyol. 2015;13(3):599-606.

Junior AV, Corcini CD, Gheller SMM, Jardim RD, Lucia Jr T, Streit Jr DP, Figueiredo MRC. Use of amides as cryoprotectants in extenders for frozen sperm of tambaqui, Colossoma macropomum. Theriogenology. 2012;78(2):244-251.

Leite LV, de Oliveira FDC, Nunes LT, Nunes JF, Salmito-Vanderley CSB. Criopreservação de sêmen de tambaqui (Colossoma macropomum) com $\mathrm{ACP}\left({ }^{\circ}\right.$ adicionado de gema de ovo. Revista Brasileira de Engenharia de Pesca. 2012;6(2):23-29.

María AN, Viveiros ATM, Freitas RTF, Oliveira AV. Extenders and cryoprotectants for cooling and freezing of piracanjuba (Brycon orbignyanus) semen, an endangered Brazilian teleost fish. Aquaculture. 2006;260(1-4):298-306.

Maria AN, Azevedo HC y Carneiro PCF. 2009. Criopreservação de sêmen de peixes no contexto do agronegócio da piscicultura. Manejo e sanidade de peixes em cultivo. Macapá, AP, Embrapa Amapá, 47-63.

María AN, Azevedo HC, Santos JP, Silva CA, Carneiro PCF. Semen characterization and sperm structure of the Amazon tambaqui Colossoma macropomum. J Appl Ichthyol. 2010;26(5):779-783.
Maria AN, Azevedo HC y Carneiro PCF. 2011. Protocolo para criopreservação do sêmen de tambaqui (Colossoma macropomum). Embrapa Tabuleiros Costeiros-Comunicado Técnico (INFOTECA-E).

María AN, Carvalho ACM, Araújo RV, Santos JP, Carneiro PCF, Azevedo HC. Use of cryotubes for the cryopreservation of tambaqui fish semen (Colossoma macropomum). Cryobiology. 2015;70(2):109-114.

Medina-Robles VM, Velasco-Santamaría YM, Cruz-Casallas PE. Efecto del volumen de empaque sobre la tasa de congelación-descongelación y la fertilidad de semen crioconservado de yamú (Brycon amazonicus). Arch Med Vet. 2007;9(3):229-237.

Merino MC, Bonilla SP, Bages F. 2013. Diagnóstico del estado de la acuicultura en Colombia. Plan Nacional de Desarrollo de la Acuicultura Sostenible en Colombia AUNAP-FAO. Bogotá, Colombia: Ministerio de Agricultura y Desarrollo Rural

Miranda LA, Chalde T, Elisio M, Strüssmann CA. Effects of global warming on fish reproductive endocrine axis, with special emphasis in pejerrey Odontesthes bonariensis. Gen Comp Endocrinol. 2013;192:45-54.

Oliveira M, Silva de Almeida-Monteiro P, Teixeira-Nunes L, AragãoLinhares FR, Silva-Pinheiro JP, Ribeiro-Pinheiro RR, Brito Salmito-Vanderley CS. Cryopreservation of tambaqui semen using a dry shipper and a programmed freezing machine. Semin Ciênc Agrár. 2016;37(4):2167-2179.

Poleo G, Aranbarrio JV, Mendoza L, Romero O. Cultivo de cachama blanca en altas densidades y en dos sistemas cerrados. Pesqui Agropecu Bras. 2011;46(4):429-437.

Ramírez-Merlano JA, Medina-Robles VM, Cruz-Casallas PE. Crioconservación seminal de bagre rayado Pseudoplatystoma metaense (Teleostei, Pimelodidae), bajo diferentes protocolos de congelación. Arch Med Vet. 2011;43:135-144.

Ramírez-Merlano JA, Velasco-Santamaría YM, Medina-Robles VM, Cruz-Casallas PE. Cryopreservation effects on the sperm quality of Cachama Blanca Piaractus brachypomus (Cuvier 1818). Aquac. Res. 2011;42(6):738-745.

Sanabria A. 2004. Catálogo de las principales especies de peces ornamentales de Colombia especies de interés comercial. INCODER, Instituto Colombiano de Desarrollo Rural.

Varela Junior AS, Corcini CD, Streit Junior DP, Rizzoto G, Jardim RD, Lucia Junior T, Figueiredo MRC. Efeito crioprotetor de diferentes concentrações do dimetilsulfóxido no congelamento de sêmen de tambaqui Colossoma macropomum. Atlantica Rio Grande. 2012;34(2):129-137.

Vieira MJAF, Carvalho MAM, Salmito-Vanderley CSB, Salgueiro CDM, Viveiros ATM, Moura AAAN, Nunes JF. Características do sêmen de tambaqui (Colossoma macropomum) em latitude equatorial. Arch Zootec. 2011;60(232):1263-1270. 